\section{Research News}

\section{Imprinting sample, please}

Taking methyl groups off a gene can be dangerous: in the case of IGF2 (insulin-like growth factor), loss of imprinting can lead to inappropriate gene activation and cancer. Now, Cui et al. turn this knowledge into an assay that could be used to predict risk for colon cancer. They had previously shown that loss of IGF2 imprinting in colon tissue occurred in $30 \%$ of patients with colon cancer. In the 14 March issue of Science, they went for the blood, examining imprinting in lymphocytes of 172 colonoscopy patients. They found that the IGF2 gene showed loss of imprinting in cells of $28 \%$ of patients with a family history of colon cancer. Although the sample group was small, loss of imprinting also occurred in $56 \%$ of those with a personal history of colon cancer. All individuals with imprinting defects in blood cells also had imprinting defects in the colon. Twenty-one patients showed loss of imprinting only in the colon but, curiously, these patients had no statistically significant history of colorectal cancer. The investigators are eager to test children to determine when such imprinting defects arise.

\section{Pancreas patch}

Bone marrow cells can give rise to pancreatic cells when transplanted into mice, providing hope for the long-term prospects of the approach for diabetes therapy, report Ianus et al. in the 13 March Journal of Clinical Investigation. Inadequate mass of pancreatic $\beta$-cell cells occurs in both type 1 and 2 diabetes. The investigators found that donor marrow cells transplanted into mice irradiated to remove endogenous marrow looked and acted a lot like $\beta$-cells. They expressed not only insulin, but also transcription factors specific for pancreatic $\beta$-cells. The investigators ruled out that cell fusion had occurred, a common criticism of such findings, by using the Cre-loxP recombination system. Different elements of the system were engineered into donor and recipient cells so that if cells fused a reporter gene would turn on; this did not occur. Pinning down the cell type in bone marrow that gives rise to pancreas should aid efforts at ex vivo expansion, differentiation, and transplantation. Malleable marrow cell types include the recently described multipotential adult progenitor cell, known to morph into the epithelium of liver, lung and gut.

\section{Well preserved}

Organ transplantation is a stressful business. Oxidative damage can cause thrombosis, organ damage and, in some cases, transplant failure. In the 1 April issue of Nature Biotechnology, Kozower et al. take on the problem of oxidative stress in a rat model of lung transplantation by treating lungs with a modified version of catalase, an antioxidant enzyme. Catalase circulates in the bloodstream and has an extremely short half-life, so it

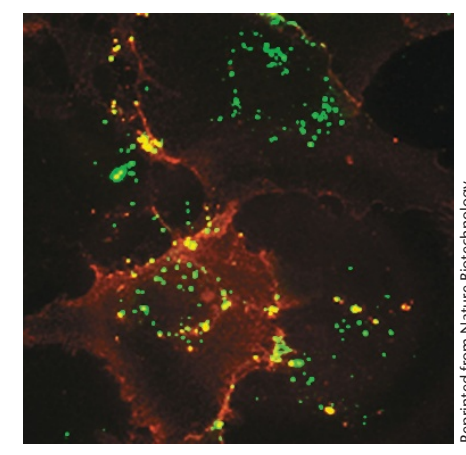

to an antibody against a protein highly expressed on endothelial cells, platelet-endothelial cell adhesion molecule-1. The chimeric catalase eases into cells within ten minutes after injection into rats-fast enough to consider practical use in organ donors. The molecule also reduced oxidative stress, ameliorated acute lung injury after transplantation and improved lung-graft function. And it worked best when given to the organ donor, not the recipient. The method also kept the lungs fresher in doesn't work well as a therapeutic agent by itself. To give catalase a boost, the investigators had to get it into the cells. They took a cue from other researchers who conjugated their enzymes of choice cold storage, allowing more time between removal and successful transplantation. The approach has the potential to work in other organs such as heart and kidney.

\section{Fat gene shows its weight}

Mutations in the gene encoding the melanocortin-4 receptor account for most known cases of genetically linked severe childhood obesity, Farooqi et al. report in the 20 March New England Journal of Medicine. And, in a match between human and test-tube data, activity of the receptor correlated with severity of disease: the individuals with the most active receptors ate the least food. Of 500 subjects with childhood obesity, $5.8 \%$ had pathogenic mutations in the gene; as children, they had consumed on average three times as much food energy than their unaffected siblings, with intake dropping off with age. All homozygous individuals were severely obese, but only $68 \%$ of heterozygotes had early-onset obesity, pointing to the influence of environmental or genetic modifiers. The receptor, a seven-transmembrane $G$ protein-coupled receptor, is expressed in the brain, mainly in the hypothalamus. The receptor receives signals from a hormone involved in the signaling of leptin, which is released by fat cells.

\section{Binding amyloid- $\beta$}

Ibuprofen and certain other nonsteroidal anti-inflammatory drugs (NSAIDs) seem to thwart Alzheimer disease, according to epidemiological studies. In the 31 March issue of Neuroscience, Agdeppa et al. add fuel to the argument that such drugs might work by doing more than counteracting inflammation. They provide

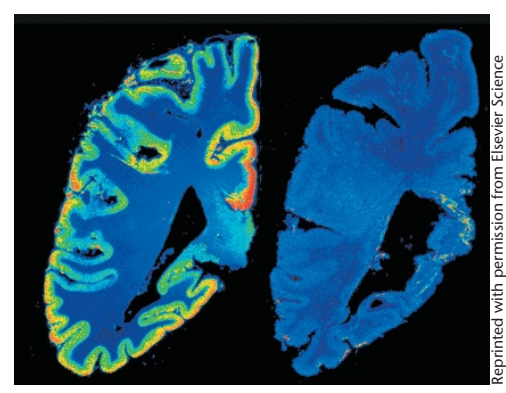
that NSAIDs and the probe appeared to share an $A \beta$ binding site. In brain slices from an Alzheimer patient, NSAIDs were evidence that certain NSAIDs bind directly to amyloid- $\beta(A \beta)$ aggregates in the brains of patients with Alzheimer disease. The authors took advantage of an imaging probe that binds $A \beta$, and found in test-tube competition experiments able to prevent binding of the probe to $\mathrm{A} \beta$ (left, probe only; right, with NSAID). Both the probe and NSAIDs prevented $A \beta$ aggregation in a test tube. These results are in line with work in cells showing inflammationindependent effects of NSAIDS on A $\beta$. Results from both types of experiments apply only to certain NSAIDs-perhaps those with the greatest potential clinical value.

Charlotte Schubert 